\title{
Photographie et représentation de soi dans $W$ ou le Souvenir d'enfance de Georges Perec
}

Siriki OUATTARA, Université Félix Houphouet Boigny, Côte d’Ivoire

Aujourd'hui la linéarité du récit fictionnel, de l'autofiction et même du journal intime ${ }^{1}$ est disloquée, voire malmenée. Selon plusieurs écrivains et critiques littéraires ${ }^{2}$, ce fait résulte de l'incapacité du récit unitaire à traduire efficacement l'objet d'une pensée. En réalité, les écrivains ont de plus en plus recours à une écriture de l'hybridité et de l'impureté au sens où Guy Scarpetta ${ }^{3}$ entend ces termes dans L'impureté. Cette nouvelle stratégie scripturale vise fondamentalement à remplacer le réalisme traditionnel fondé sur une vraisemblance mimétique du sociopolitique, voire du culturel, par un nouveau réalisme qui privilégie une représentation dynamique de l'histoire et où il est question de construction du sens dispersé dans le récit, lui-même fragmenté, disloqué.

$W$ ou le souvenir d'enfance convoque ouvertement en son sein des éléments paralittéraires comme la photographie qui le déconstruit. Le désir de Georges Perec de reconstituer ou de reconstruire son histoire est si ardent qu'il lui a consacré ce roman particulier. Dans cette œuvre autobiographique atypique ${ }^{4}$, l'auteur fait appel à diverses techniques de représentation de soi $^{5}$, la photographie. Cette dernière est un élément nouveau en littérature (même s'elle y est prise en compte depuis le dix-neuvième siècle) qui redéfinit nombre d'habitudes littéraires. Ainsi, elle occasionne un renouvellement de l'écriture à travers l'institution de nouveaux rapports qui, tout en changeant les vieux rôles narratifs, invitent à dire autrement, voire à raconter différemment. La photographie offre alors l'occasion d'expérimenter une nouvelle discursivité de la représentation.

Ma contribution se propose de montrer en premier lieu la particularité de ce dispositif narratif - la représentation rétro-prospective - que l'insertion de cet élément paratextuel dans le récit impose au texte en se fondant sur les travaux de Lucien Dällenbach. En fait, cette technique est un aspect de la mise en abyme qui donne à Perec l'occasion d'effectuer l'enchâssement de diverses représentations parfois contradictoires d'un même fait ou évènement. En second lieu, sous l'autorité des écrits théoriques de Jean-Marie Kouakou, elle analysera les relations entre la photographie et la notion de perception pour mener une enquête sur soi et, en dernier lieu, elle révèle la manière dont Perec s'adonne à un travail d'auto-engendrement par le biais d'un certain usage de la photographie. 
On sait depuis les travaux de Zeynep Mennan (46) et même bien avant que le début de la vie de Perec est marqué par un trou de mémoire, une absence d'histoire due à la mort de ses géniteurs. Aussi l'écriture photographique offre-t-elle à ce dernier l'occasion de combler ce vide par sa volonté de faire revivre le passé en rappelant certains souvenirs lointains et de mettre au jour des traces et des vies perdues. En effet, Perec est convaincu que seule l'écriture peut lui permettre de surmonter cette absence et de redonner une seconde vie aux disparus. Cette observation faite par Elizabeth Molkou dans son analyse de Au-delà de l'autofiction de Pierre Lepape à propos de l'autofiction semble caractériser l'entreprise de Perec dans son livre. Elle avance justement qu' «écrire après la Shoah implique pour tous ces écrivains l'impossibilité d'un recours à des formes narratives traditionnelles » (147-149). C'est fort de cette réalité que, en 1975, Perec écrit ce roman accordant une importance aux photographies des siens dans l'enquête autocentrée qu'il mène pour refaire le chemin qu'il a déjà parcouru enfant. Un usage particulier de la photographie permettra à cet écrivain de représenter son histoire qu'il n'a pas connue.

\section{La photographie : une représentation rétro-prospective}

Dans «Intertexte et autotexte », Lucien Dällenbach répertorie trois types de mise en abyme en tenant compte de la notion de chronologie dans le récit. Il y note que « la 3 , rétroprospective, réfléchit l'histoire en découvrant les événements antérieurs et les événements postérieurs à son point d'ancrage dans le récit » (287). La mise en abyme rétro-prospective, en fonction de son emplacement intermédiaire voire médian, serait, dans ces conditions, la « charnière entre un déjà et un pas encore, celle-ci non seulement combine les vecteurs temporels et les fonctions des réflexions précédentes ; (...) elle possède une économie propre toute entière sur l'extrapolation » (289). En fait, cette technique dévoile le rapport que Perec entretient avec le document photographique. La relation de cet auteur à la photo, justement, «s'effectue littéralement sous le signe d'un ça a été irréfutable et d'un momento mori qui invitent à reconstituer non seulement la scène représentée, mais aussi ce dont elle marque le point d'aboutissement» (Dällenbach, Simon, 55). Il y a donc, en la photographie, une compétence à, non seulement stimuler l'imagination, mais surtout à faire démarrer significativement l'acte même d'écriture (150).

L'usage de la photographie, élément paratextuel, dans la narration de $W$ ou le souvenir d'enfance, en sa partie autobiographique, semble obéir à cette technique scripturale et semble répondre au besoin de Perec de donner à son texte une valeur historique qui induit de facto une solidité autobiographique au roman. Dans $W$ ou le souvenir d'enfance, la vue des 
photographies $^{6}$ des géniteurs du narrateur-voyeur engendre un deuxième récit, descriptif, de ces images (qui sont uniquement mentales; on ne voit pas les photos dans l'œuvre) puis un troisième récit, explicatif cette fois, en rapport direct avec elles. De fait, l'œil qui capte premièrement l'image est largement ignoré. Il serait alors plus juste de parler d'un ensemble de fragments de textes se résumant en des lignes de proportions diverses, des sortes de résurgence de 1'histoire personnelle du narrateur en quête de son passé familial. Ces récits descriptifs, remplaçant les photographies, ambitionnent de conserver intacts certains souvenirs chers à Perec.

La constance qui se dégage de ces occurrences, c'est que le texte et le narrateur-voyeur évitent soigneusement d'établir une relation directe entre l'œil du ou des photographe(s) et les objets anthropomorphes photographiés. Il y a un refus de leur accorder même la faculté et donc la possibilité de regarder. Ce procédé peut bien traduire la volonté du narrateur autobiographique de détenir le rôle exclusif de témoigner le passé des siens. En ce cas, et le plus logiquement possible, le « regard [de ce narrateur autobiographique doit être] qualifiant vis-à-vis [de l'] objet qui reste marqué par la forme passive du vu exclusif, et qualifié donc » (Kouakou 151).

Cependant, c'est bien par la photographie, produit du travail du premier œil-voyeur (celui du photographe), qu'est initié le premier niveau de la mise en abyme. Sa description par le narrateur-enfant constitue le second niveau (46) et les notes de rectification qui la déterminent, sous forme critique du narrateur-adulte, indiquent le troisième niveau de la mise en pratique de la technique. Justement, par cette mise en abyme, Perec donne à son texte une objectivité historique. Pour cela, le narrateur devenu adulte n'hésite pas à revisiter tous ses souvenirs enfantins, à les scruter, les critiquer et il va même jusqu'à mettre en doute leur fiabilité (53-62). Par un tel fait, il s'adonne à une dénonciation des ruses que lui joua sa propre mémoire; adoptant ainsi une position hypercritique. L'adoption de cette technique de dédoublement du narrateur/auteur laisse transparaitre que $W$ ou le souvenir d'enfance remet en cause le statut divin de l'écrivain en le relativisant à travers une inscription en son sein des contestations des narrataires. C'est le cas de Perec adulte qui ne partage plus les propos de Perec enfant. Mais, davantage encore, un tel type de narrataire dans l'imposant appareil des notes au huitième chapitre introduit dans le livre la technique de la déconstruction, un aspect de la mise en abyme.

En fait, il s'agit d'un texte qui met en abyme le second, celui fait par le narrateur-enfant, c'est-à-dire celui des souvenirs de ce voyeur. Il lui fait, par conséquent, écho. Le texte des souvenirs du narrateur-enfant défigure le premier et accroit la difficulté de lecture parce qu'il 
force le lecteur à interrompre la linéarité de sa lecture ${ }^{7}$ mais il complète également ce deuxième texte, l'explique, le commente, puisqu'il est rattaché à lui. Tout se passe alors comme s'il y avait, chez ce descripteur, une sorte de retour du refoulé, une sorte de remémoration due à ce que l'œil voit ou est en train de voir.

Cette possibilité de revivre les moments passés que la photo accorde au voyeur, implique forcément une simultanéité entre l'objet regardé (l'acte de regarder) et la remémoration. Il ne serait pas faux dans cette logique de soutenir que, en plus de faciliter et de permettre le souvenir, l'œil ou précisément l'acte de regarder la photographie chez Perec permet surtout un travail de l'imagination. La photo doit être prise comme le ferment de l'imagination créatrice ici. Cela peut se comprendre à partir justement de cette évidence que Jean-Marie Kouakou révèle à partir de l'œuvre de Claude Simon :

L'œil, répondant au vœu du narratif qu'on doit nécessairement trouver dans le genre, a un caractère paradoxal et particulier. Chez Simon [comme chez Perec], «il ne voit pas, il se souvient»: on le fait ainsi et du coup sortir de l'universalité de la vocation originaire en tant que sens de perception. Et, comme les séquences consacrées à la description sont abondantes, l'œil contraint à une opération critique élastique qui oblige à faire la distinction synchronique entre voir et dire puisque les deux se superposent au moment de la pratique : il y a un espace de division à déterminer avec précision. (149)

Concrètement, si la photo de la mère (53) et celle du père (46) représentent dans le texte le passé (anachronie) de Perec, et l'œil-voyeur du narrateur représente le présent de narration, l'histoire qui découle de la vue de la photographie est à la fois passée, présente et future parce que le narrateur tient compte du contexte circonstanciel et même historico-événementiel de la prise de cette image et la postérité qui appréciera ses commentaires.

L'importante quantité de notes infrapaginales (vingt-six en tout) de la page 53 à la page $62 \mathrm{du}$ roman trouve alors sa justification dans la recherche de l'objectivité, de l'impartialité au nom de la vérité historique ${ }^{8}$. Dällenbach précise du reste que

Sous la juridiction du contexte qui la précède, la réflexion rétro-prospective peut faire retour sur lui, lui surajouter son sens et agir sur la suite du texte, désormais sous sa juridiction thématique. Présupposée et présupposante, objet et sujet d'interprétation, elle trouve en ce site la plate-forme qu'elle recherche pour faire pivoter la lecture ( $«$ Texte », 290)

De la sorte, il y a obligatoirement une mise en scène à partir de la photographie par le biais du texte bref qui fragmente le récit initial et par les notes qui rectifient le premier commentaire, celui du narrateur-enfant qui « est fait de pseudo vérité, d'affabulation et de fantasme. C'est une histoire décousue, fausse faite de vide, qui ne possède pas la solidité, la consistance du récit autobiographique » (Sérifou 166). Il faut finalement admettre que dans ce 
livre, le personnage-narrateur qui regarde les photos est à considérer comme un point de vue dont le foyer focal est réglé selon le vecteur œil $\rightarrow$ mémoire.

Contrairement à ce qu'Adélaïde Bakissia Sérifou écrit au sujet des Choses, dans $W$ ou le souvenir d'enfance, le mouvement de la narration part de l'œil vers la mémoire. Ici, le processus implique forcément la vue d'abord de l'objet (la photo) qui, à son tour, crée le déclic de la narration à partir de la mémoire (le souvenir). Ainsi, le narrateur fait découvrir les souvenirs du sujet-percepteur de la photographie. Dans une telle logique, l'univers fictionnel du livre est découvert à partir, bien évidemment, non de la conscience, mais de l'inconscient d'où il y a un retour fort impressionnant du refoulé ; nous dirons bien volontiers du déjà-vu. Cette autre disposition amène à voir l'œil comme un véritable condensateur des faits vécus, passés. C'est donc un œil qui n'oublie pas, un œil-mémoire?

Pour comprendre davantage ce mécanisme scriptural mis en place dans ce livre, relisons une fois de plus Dällenbach qui nous le rappelle, certes à propos de Claude Simon mais son explication éclaire la stratégie narrative adoptée par Perec quand il mentionne le mouvement du regard sur la photo :

L'œil qui observe se mue [...] en œil qui épie et qui se perd dans le spectacle, la fascination le faisant passer de l'autre côté du miroir. Traitée d'abord en focalisation externe et respectueuse du signifiant photographique, la description vire progressivement, palier par palier, à la description à focalisation interne qui déploie (ou développe) l'image simultanée en récit -en récit filmique pourrait-on dire. (Simon, 55-56)

Le texte produit à partir du regard posé sur les photographies ${ }^{10}$ fait découvrir la présence de deux narrateurs, une sorte de dédoublement du narrateur principal : l'un qui est rattaché au passé, aux souvenirs qu'il relate (Le narrateur-enfant) et l'autre, le narrateur-adulte, qui fait œuvre de devoir de mémoire. On comprend peut-être ainsi mieux maintenant la position défendue ici par Kouakou lorsqu'il affirme que:

La procédure est très souvent volontairement (?) anachronique. Ici, la scène est le plus souvent décalée et passée. Ce que le voyeur observe c'est ce qui a déjà été vu et saisi une première fois par un artiste : les cartes postales, les photographies, les timbres, etc. Le voyeur se remémore donc, il voit une deuxième fois. Cela suppose qu'il y a transfocalisation dans la mesure où il voit voir, il voit dans les yeux de quelqu'un d'autre; et il y a métafocalisation dans la mesure où (la métaphore de) la profondeur sonde lui permet d'aller audelà du visible par libération (79)

Le texte qu'il produit n'est que la re-présentation de ce qu'il «voit dans sa tête », en pensée, une représentation mentale donc à la vue des photos de ses géniteurs. Il y a bien, en ce cas, une projection analepsique (une projection par anachronie) du narrateur qui tente, par cet 
exercice, de saisir la réalité, mieux l'exactitude des faits liés à ces images. C'est-à-dire que Perec tente par le canal des photos de ses parents de

raconter une période décisive de sa vie marquée par un vide décisif, celui de la perte de ses parents, qui a associé la découverte de sa vocation d'écrivain. Parler des souvenirs mineurs qu'il remémore lui permettant d'une nouvelle façon de redonner sens à sa vie, l'absence d'histoire et de trace sera l'élément phare qui le poussera à écrire. Ainsi l'écriture [photographique] devient un moyen de retrouver le parcours de son enfance. (Mennan 48)

On n'est évidemment pas loin ici du studium et du punctum ${ }^{11}$ dont parle Roland Barthes dans La chambre claire. Dans la narration du texte, cette production constitue une sorte de cicatrice dans le texte principal à l'intérieur duquel il se situe puisqu'il rompt la nappe de la narration en contestant un récit unitaire, disloque le lisse de la continuité en multipliant et divisant les réflexions, ce qui voue, comme par fatalité, le récit à l'éparpillement. Ainsi, ce second texte empêche l'établissement d'un sens linéaire, le dissémine à partir d'un éclatement des phrases et autres stratégies. Régine Robin souligne que la technique déplace et déconstruit tout « ce par quoi 'le biographique' fait texte» (10), en d'autres termes, « récit fragmentaire de l'enfance, souvenirs, notules et historiettes, formations » (10). La conséquence de cette pratique est la transgression des conventions autobiographiques et narratives.

La photographie, telle qu'elle est utilisée dans ce « roman atypique » de Perec, en sa technique et en tant que dispositif, est à considérer comme une technique de mise en abyme (autotexte). L'écrivain l'utilise pour revenir sur un pan de son histoire familiale car,

l'impression de bougé permet non seulement de restituer le mouvement des personnages avant et après l'instantané et, à la faveur de la synesthésie, de faire revivre pour un instant ceux qui sont morts $^{12}$; à force d'interroger la photo jaunie et d'insuffler vie à ce fossile, le sujet qui l'anime et qu'elle anime finit par l'annuler comme médium et par entrer lui-même en scène. (Dällenbach, "Texte", 56)

Mais, quelle lecture de la perception peut-on faire de cet élément paralittéraire dans la quête de soi du romancier?

\section{Photographie et perception}

L'objet observé, c'est-à-dire l'objet référent, en l'occurrence les parents de Perec qui sont des objets anthropomorphes, n'est pas directement regardé par le scripteur. Ce dernier accomplit un travail second car travaillant sur un déjà-là. Par conséquent, Il s'agit d'une représentation des parents : l'image décrite, présentée est bien ce que le foyer percepteur enfant transmet. La technicité d'une telle entreprise ressemble en plusieurs de ses points à ce que Kouakou décrit dans La chose littéraire lorsqu'il analyse les rapports du voyeur aux objets 
regardés. Son étude sur la question de distance entre ces deux pôles éclaire la stratégie de Georges Perec dans $W$ ou le souvenir d'enfance dans la mesure où il soutient que

le regard se pose surtout sur l'invisible. Il se tient alors à distance respectable du visible car ce qui l'intéresse, c'est l'au-delà de la surface pouvant le conduire vers un imaginaire parfois fantasmagorique et non pas l'objet en luimême qui, de toute façon, reste inconnu. Ainsi par exemple, chez Perec, [...] ce qui reste, c'est le fond d'invisibilité: l'œil joue ainsi à liquéfier progressivement ce qu'il voit. (167)

Dans une telle perspective, on débouche inévitablement sur autre chose que la photographie regardée. Tout conduit à observer sa liquéfaction, son délaissement au profit d'une histoire dont elle demeure l'élément déclencheur, le fondement. En ce cas, l'image photographique fonctionne tel un «négatif fécond».

Mais, dans le roman étudié, Perec complexifie davantage le procédé avec l'entrée en scène d'un deuxième narrateur qui est un critique. Le texte qu'il produit - et qui est en notes infrapaginales — «abyme » fortement l'objet regardé. Dans ce cas, il y a bien plus qu'une représentation : on doit plutôt parler de re-re-présentation puisque l'objet perçu (les géniteurs de Perec) est revisité trois fois : par le photographe d'abord, ensuite par le narrateur-enfant et enfin par le même narrateur devenu adulte. Les diverses perceptions se réalisent chaque fois à partir du travail effectué par un autre premier. Ainsi, les révélations du narrateur-enfant ont pour base les images fixées par le(s) photographe(s) quand le texte du narrateur-adulte a pour fondement le discours produit par lui-même lorsqu'il était encore enfant sur ces mêmes photos. Les notes infrapaginales critiquent, en fait, le déjà-réalisé de Perec-enfant. Dans ces conditions et vu la divergence qui ressort de ces positions, l'on note forcément un problème au niveau de la fiabilité des discours ou informations données sur l'objet regardé par les deux derniers foyers percepteurs ${ }^{13}$. L'on note, de toute évidence, un dédoublement du narrateur qui minimise de facto toute notion de distance. Dans le roman, on ne voit jamais les parents déjà morts. Seules leurs images sont commentées par le voyeur quand il était encore enfant :

l'auteur $[\ldots]$ tente à partir de quelques photos jaunies, de souvenirs lacunaires et douteux, de restituer la trame de sa petite enfance. En dépit de la défaillance de sa mémoire dépourvue de repères tangibles, il ressent cruellement le besoin de se conformer à son enfance assassinée par «l'Histoire avec sa grande hache » et recourt aux mémoires des autres, de ses proches, à leurs témoignages pour vérifier les images de sa mémoire. (Mennan 52)

Mais dans le discours descriptif fait par le narrateur adulte sur son travail fait jadis, on lit bien que l'image n'est plus devant mais en lui. Ce procédé amène alors à soutenir que :

L'écart physique est comblé par l'impossible possibilité de situer le percepteur et le perçu comme deux points (au sens géométrique) distincts. Les deux se 
restituent l'un dans l'autre, l'un à partir de l'autre du fait de la parole qui est toujours actualisée c'est-à-dire présente et qui rend possible, au moment de son expression, l'existence des deux étants. (Kouakou 169)

Au-delà, le texte instaure, par l'acceptation de ces différents niveaux de perception en son sein, un dialogue avec un autre voyeur placé hors de la diégèse. Il revient à ce dernier de faire une analyse de tous ces objets littéraires ainsi que des divers discours produits sur chacun d'eux afin de situer l'intention de l'auteur c'est-à-dire le non-dit de ce texte. Cet autre percepteur-critique, Kouakou le nomme «le lecteur concret» (170). En clair, le narrateur, ainsi que le photographe, qui sont des objets anthropomorphes, sont alors pris comme des « objets littéraires en tant que faits de récits» (170). De telles observations approfondissent l'analyse en mettant un accent sur les investigations effectuées par Perec-narrateur/enquêteur pour se dire. En conséquence, on est dans la logique d'une enquête visant à découvrir, non un coupable, mais bien un pan de vie, une histoire propre à ce narrateur autobiographique.

Pour revenir à la photographie à proprement dit, soulignons que le « regard-sonde» (Dällenbach) que Kouakou nomme «profondeur-sonde» posé par le voyeur sur la photographie donne au sujet percepteur la faculté de redonner vie aux morts grâce à son imagination. Dans $W$ ou le souvenir d'enfance, Perec expérimente cette autre possibilité offerte par la photo pour retrouver la saveur d'une vie familiale qu'il n'a pas vraiment connue(s) et qu'il recrée ici.

\section{La reconstruction de l'arbre généalogique à partir de la photographie}

Quand Mennan affirme que la vie de Perec débute par un vide, un trou que le romancier entend combler avec la fiction à partir de laquelle il se recrée un foyer, son lecteur a tendance à ignorer le grand apport de la photographie dans l'accomplissement de cet objectif. $W$ ou le souvenir d'enfance revient sur les grandes étapes de la vie de son auteur en empruntant le canal d'une certaine écriture littérarisée de la photo, celle donc « où se trouve 'inscrit et décrit' le chemin parcouru par l'auteur» (46). En clair, nous verrons que la photo permet à ce romancier de représenter son histoire.

Perec voit, en effet, en la photographie un moyen pour redonner vie aux siens ${ }^{14}$. En fait, il la considère comme un élément qui l'aidera à faire éclore sa mémoire familiale et même juive. Ce qui s'avère d'ailleurs juste dans $W$, si l'on s'en tient strictement à ce qui est dit dans la note numéro 8. L'auteur y fixe l'arbre généalogique des Perec (56-57). L'insertion de cette généalogie auctoriale remontant aux origines même de son nom dans ce roman est en réalité une tentative d'explication à laquelle il s'adonne à partir des deux photographies du père (46). 
Au-delà de la « résurrection » de ses parents paternels dans cette note, c'est tout l'exode effectué par sa famille juive depuis l'Espagne où « les Peretz font volontiers remonter leur origine » (56) qu'il fictionnalise ici.

Ainsi, la photographie du père offre l'occasion de créer une histoire. On assiste alors à une sorte de restitution historique dont l'Histoire l'en a privé. Un tel traitement de ce modèle photographique fait prendre conscience que la photo fonctionne comme un déclencheur de fiction dans laquelle la narration s'apparente à une sorte de recherches effectuées sur les origines de son créateur. On peut considérer que la photographie est pour Perec une archive privée,

essentiellement familiale et à strict usage personnel, elle [la photo] concerne bien le sujet, mais dans un autre (que) lui-même: un proche dont ce sujet est irrémédiablement coupé et dont le passé (un certain passé en tout cas) lui demeure insu, l'archive s'avéra la seule voie d'accès, d'ailleurs toute conjecturale, à cette marge d'inconnu qu'il importe précisément de réduire (Dällenbach, Simon, 55).

Il en va de même de celle de la génitrice du romancier dans le roman. Perec lui rend un hommage spécial en y imposant son image de manière éternelle. De la sorte, il se venge de la vie réelle en créant un monde fictionnel, à partir surtout de la photographie, dans lequel sa mère reste éternelle. De la note treize à la note vingt-six, le romancier revient sur la vie de sa génitrice et de toute sa famille. La résurgence des souvenirs, de l'histoire de sa branche maternelle commande le gros plan sur sa déportation et sa disparition. En effet, dans la note vingt-six, il donne les raisons de la dite déportation (53).

Arrêtée avec sa sœur malgré son titre de veuve de guerre lors d'une rafle, toutes les deux détenues à Drancy le 23 janvier 1943, Cécile Perec et sa sœur prirent le chemin d'Auschwitz le 11 février de la même année. La technique adoptée dans $W$ ou le souvenir d'enfance permet de tirer de la photographie des récits où il est question de recherches sur le passé du voyeur-narrateur, d'interrogations sur la vie de la famille du romancier à ressusciter. Un tel procédé est bien proche de la technique totipotentielle qu'évoque Élizabeth Bizouard ${ }^{15}$.

Cette approche fait de Perec un véritable archéologue enquêtant sur une vie communautaire antérieure à faire revivre. En effet, ici, toute son «opération implique des stratégies et des plans de recherche et ressemble fortement aux efforts d'un paléontologue qui, à partir de quelques fragments d'os, reconstitue un animal complet » (Kostrubiec 20). Il y aurait là, apparemment, une volonté de cet auteur de témoigner et de relater, par le biais de l'écriture photographique, l'histoire de ses géniteurs et celle des les autres disparus de sa communauté d'origine. Timo Obergöker aboutit à une conclusion semblable quand il analyse la question de la préservation de la mémoire chez Perec. Dans le sous chapitre « Une mémoire 
quotidienne », il souligne que la photographie constitue le pont entre le sujet-percepteur et sa famille. De la sorte, la mémoire visible incarnée par la photographie crée des repères visibles qui le maintiennent, le fixent dans une généalogie rassurante. La souffrance de Perecpercepteur se résume uniquement en l'absence des siens (328).

En somme, une telle enquête sur ses origines est une véritable lutte contre l'oubli, un réel combat contre l'absence, le vide. En ce cas, la photographie fait revivre ce qui a été, ce qui est fini, accompli et que Perec n'a pas vraiment vécu. Elle l'autorise à revisiter son passé. Le récit des investigations mémorielles effectuées à la suite de la vue de l'image photographique est bien une entreprise dans laquelle les souvenirs constituent les matériaux de reconstruction et de reconstitution des connaissances consignées dans le récit d'enquête réflexive ainsi élaboré. L'usage de la photo dans son livre répond, à bien des égards, à un besoin de l'écrivain de dire l'ineffable, une sorte de conjuration de l'absence en ressuscitant les siens et leur histoire.

\section{Bibliographie}

Barthes, Roland. La chambre claire : note sur la photographie. Paris : Gallimard/Seuil, 1980.

Bizouard, Elizabeth. Le cinquième fantasme: auto-engendrement et impulsion créatrice. Paris : PUF, 1995.

Ceccon, Jérôme. "Ghislaine Charlier et Jan-jan Dominique : récit culturel d'un passé mémoriel. Le souvenir : formes et mutations de la mémoire culturelles haïtiennes des femmes écrivains de la diaspora». Mémoire et culture : actes du colloque international de Limoges. 10-12 décembre 2003. Limoges : PULIM, 2006.

Chartier, Pierre. Introduction aux grandes théories du roman. Paris : Bordas, 1990.

Coulibaly, Adama. «La pratique postmoderne dans l'écriture romanesque de quelques écrivains francophones d'Afrique Noire ». Thèse. U de Cocody, 2007.

Dällenbach, Lucien, « Texte et autotexte ». Poétique 27 (1976).

---. Claude Simon. Paris : Les contemplations / Seuil, 1988.

Foucault, Michel. «Qu'est-ce qu'un auteur?». Dits et écrits. Vol. 1. Paris : Gallimard, 1994.

Gascar, Pierre. Préface. L'Inde ou l'Amérique. Pierre Mertens. Paris : Seuil, 1969 ; Ancre rouge, 1999.

Kofman, Sarah. L'enfance de l'art. Une interprétation de l'esthétique freudienne. Paris : Galilée, 1985.

Kostrubiec, Viviane. La mémoire émergente: vers une approche dynamique de la mémorisation. Paris : Harmattan, 2001. 
Kouakou, Jean-Marie. La chose littéraire, objet/objets. Abidjan : EDUCI, 2005.

Mennan, Zeynep. «Une autobiographie atypique : $W$ ou le souvenir d'enfance de Georges Perec ». http://www.edebiyatdergisi.hacettepe.edu..tr/2006231ZeynepMennan.pdf, Edebiyat Fakültes Dergisi Cilt : 23, Say1 : 1, Haziran 2006. 45-55.

Molkou, Elizabeth. «L'autofiction : un genre frontière». Ecrire la frontière. Limoges : PUL, 2003

Obergöker, Timo. Ecriture du non-lieu. Topographie d'une Impossible quête identitaire: Romain Gary, Patrick Modiano et Georges Perec. Francfort : 2004.

Perec, Georges. W ou le souvenir d'enfance. Paris : Gallimard/Denoë1/L'imaginaire, 1975.

Ribaupierre, Claire de. Le roman généalogique : Claude Simon et Georges Perec. Bruxelles : Part de l'œil, 2002.

Robin, Régine. Le golem de l'écriture : De l'autofiction au cybersoi. Montréal : XYZ, 1998.

Sartre, Jean-Paul. « M. Mauriac et le roman ». Situation I. Paris : Gallimard, 1947.

Scarpetta, Guy. L'impureté. Paris : Grasset et Fasquelle, 1985.

Skutta, Franciska. "Et si on écrivait des photos ? A propos de Tardifs instantanés de Jean-Loup Trassard ». Narratologie 6 (2005) : 365-376.

Sérifou, Adélaïde Bakissia. «Procédés de création et supports de l'imagination chez Georges Perec ». Thèse. U de Cocody-Abidjan.

\footnotetext{
NOTES

${ }^{1}$ Le journal intime offre un texte dans lequel le Moi est inévitablement fragmenté à la fois par le silence et le sommeil.

2 Dans Creuser l'émiettement, construire le morcellement (à partir de vies potentielles), Paris, Seuil, 2001, Claude Burgelin revient sur la tentative de représentation des effondrements du vingtième siècle par Camille de Toledo. A partir de quarante-sept petites histoires construites autour de vies morcelées, fissurées, explosées, l'auteur témoigne dans ses récits d'une expérience psychique d'implosion, d'effraction, de l'inquiétude d'être au monde. Sepp Arvi, lui, étudie la question dans «L'écriture insulaire. Pour une poétique du fragment dans le journal intime ", La revue du CRELIS, 2012. Roland Barthes, dans Roland Barthes par Roland Barthes s'adonne à une sorte d'autoportrait atypique où le respect d'un ordre alphabétique des thèmes assure une dimension fragmentaire à la composition de son récit. À cela s'ajoute l'influence de la conception nietszchéenne du Moi sur les écrivains comme Henri Michaux avec son Moi « foule».

${ }^{3}$ Pour Guy Scarpetta, l'impureté consiste en un mélange des genres et des registres par ce que dans son entendement, aucun art n'est homogène, naturel. Par conséquent, l'œuvre d'art en général et l'œuvre littéraire se présente tel un patchwork.

${ }^{4}$ On lira avec intérêt l'article de Zeynep Mennan intitulé « une autobiographie atypique : $W$ ou le souvenir d'enfance de Georges Perec », 45-55.

${ }^{5}$ Cette affirmation dévoile tout l'écart qui sépare Perec d'une certaine nouvelle conception du roman telle que Michel Foucault l'entend. Dans son article intitulé «Qu'est-ce qu'un auteur ? », 803, ce dernier avoue en effet que « dans un roman qui se présente comme le récit d'un narrateur, le pronom de première personne, le présent de l'indicatif, les signes de localisation ne renvoient jamais exactement à l'écrivain, ni au moment où il écrit ni au geste même de son écriture » (803). W ou le souvenir d'enfance se situe, en sa partie prise en charge par le Je narrant, aux antipodes de cette position défendue par Foucault. Ainsi, dans ce livre, on peut bien parler d'une renarrativisation prospérant sur le terreau d'une résurrection de l'écrivain. Avec $W$ ou le souvenir d'enfance Perec s'inscrit, ici, dans la perspective du retour à la forme traditionnelle du récit.
} 
${ }^{6}$ Notons que l'usage du verbe « voir » est, ici, quelque peu excessif. Dans le livre, « aucune image, quelle qu'elle soit, ne vient animer ou perturber la sobre typographie du volume. Il ne s'agit donc pas ici d'une présence simultanée de deux types de messages visuels, linguistique et non linguistique, et qui se conditionneraient réciproquement » (Skutta 365). La technique de Perec donne plutôt a voir, en fait, des photos génératrices de récits d'enquête réflexive. Le procédé implique ainsi la naissance d'un texte littéraire dont le fondement reste la photographie, une image absente du roman et antérieure à lui.

${ }^{7}$ Franciska Skutta renforce cette position quand elle souligne qu'une telle stratégie scripturale constitue un véritable défi à "toute idée d'une progression continue, chaque texte, telle une photo, s'offre ainsi à une contemplation plus ou moins prolongée, voire répétée, ce qui relativise le principe fondateur même du message linguistique, à savoir la linéarité de son signifiant. Ici, cependant, la linéarité brisée, et avec cela, l'émergence d'une certaine iconicité, ne se manifestent pas seulement au niveau de l'enchaînement des textes dans l'ensemble [de l'œuvre], car l'écrivain 'y invente un discours qui vise à briser toutes les continuités [...] pour cerner la solitude d'un instant, d'un lieu, d'un objet, d'un souvenir' » (366-36).

${ }^{8}$ Claire de Ribaupierre indique que dans le texte la conséquence de ce but visé par le narrateur-adulte est bien « la description des images [qui] se veut objective, neutre, précise, signalétique » (72).

${ }^{9}$ Selon les analyses d'Adélaïde Bakissia Sérifou, Cet œil-mémoire supplée le narrateur qui, à dire vrai, « n'est plus un être. Il n'est plus qu'un organe, une faculté, et le récit se fait à partir des données dont dispose cette faculté » (15-16). Pour elle, récit perecquien a trois moyens de perception : un narrateur-œil, un narrateurmémoire, un narrateur œil-mémoire » (16).

${ }^{10}$ Ici nous invitons le lecteur à considérer aussi la photographie elle-même et non uniquement le regard posé sur elle. Chez Perec, cet objet généralement conservé soigneusement est un véritable «lieu de mémoire », un condensé du passé oublié. En elle, nous dit Jérôme Ceccon parlant certes de « ce qui a été jeté » et qu'il faudra considérer comme "une archive », mais qui se vérifie aussi avec la photographie, " est contenu un texte qui commémore un tournant historique des événements et articule une vision d'équité sociale et de classe » (200).

11 Roland Barthes a créé le studium et le punctum et les oppose dans La chambre claire: note sur la photographie. Cependant, c'est Régine Robin qui fournira des définitions claires de ces termes. Dans Le golem de l'écriture : de l'autofiction au cybersoi (1998), elle affirme que « le studium, ce sont les 'bioses', les parcours obligés du cycle de vie, les grandes périodisations qui rythment les biographies : scolarité, entrée dans le monde du travail, départ du foyer parental, formation d'un foyer indépendant, mariage, naissance, mort des proches, retraite, changement de lieu d'enracinement par mariage, émigration etc. C'est aussi pour l'homme public ou pour l'écrivain, les grands événements où diverses temporalités s'entrechoquent, les dates de publication etc. [...]. Le studium, c'est l'investissement sociologique de la photo. Il est toujours codé, attendu. Il chasse l'incongru » (10). Quant au punctum, elle indique que c'est un "excès de sens, de détail qui mobilise l'affect, choc, surprise, contingence dont le sens échappe, car il ne peut d'emblée se classer, se ranger dans une catégorie conceptuelle préalable. Au punctum correspond le biographème. Non la linéarité apparente d'un trajet, mais des détails, des inflexions, une vie trouée, l'irruption de signifiants inattendus» (11). Et Skutta de dire que «le punctum est un détail dans la photo qui attire et fixe le regard, mais dont la force troublante résiste à 1'explication.- Dans la scène du théâtre, le punctum est à comprendre par rapport au narrateur-spectateur » (373).

12 Cette position de Dällenbach, Marielle Chauvin l'explicitera davantage dans « La fiction à l'épreuve de l'image fixe : représentation et dispositif. L'exemple de Jeff Wall », " Penser les représentations fictionnelles », encore inédit, sous la direction de Kouakou, quand elle révèle que « En effet, en 1839, lorsque Arago présente officiellement l'invention de Daguerre à l'académie des sciences, la photographie est montrée comme un moyen d'enregistrement fidèle du réel » (77).

${ }^{13}$ Cet aspect de la question résulte du fait que de plus en plus la photographie se met « irrémédiablement à 'mentir', au sens platonicien du terme, inventant preuves, évènements, personnages, mettant en scène un monde merveilleux » (78). Ce point de vue de Chauvin jette un éclairage sur l'analyse en faisant comprendre que la photographie peut bien être culturalisée, c'est-à-dire qu'elle peut être montrée selon la vision de son présentateur. C'est d'ailleurs ce que fait Perec enfant dans le texte. On assiste alors, dans cette logique chez Perec, au rêve enfantin de l'écrivain qu'il s'empressera de corriger une fois devenu adulte puisque, essentiellement « la photographie, dans son histoire même, feint et façonne le réel» (78).

${ }^{14}$ Sur cette conception du rôle de la photographie en littérature, nous voudrions bien rappeler ici la thèse d'Adama Coulibaly avec qui nous partageons le même avis lorsqu'il soutient, au sujet de Le lys et le flamboyant de Henri Lopes, que «La photographie s'installe au cœur de la problématique identitaire du métis dans notre corpus. Elle renvoie à la question essentielle : celle de la saisie de 1'essence des choses et des êtres », 438-439.

15 Dans Le cinquième fantasme: Auto-engendrement et impulsion créatrice, Bizouard indique que la totipotentialité est justement un processus permettant à l'écrivain, qui est un élément, un membre d'une famille, de reconstituer cette dernière. En somme, cette technique vise à reproduire toute l'ascendance de l'auteur à partir de son œuvre. Par une telle résolution (celle de reconstruire son arbre généalogique ainsi que l'histoire de celleci), Perec s'adonne à un auto-engendrement de ses fantasmes enfantins, de son histoire. Avec lui, se dévoile alors 
la certitude que «nos premières expériences du monde continuent de déterminer, en grande partie, nous le savons, notre vie mentale et les mouvements de notre sensibilité. [...] La régression infantile, retour à l'inexprimé, à la vraie source, constitue une névrose. Les souvenirs d'enfance, une consolation. Une revanche. Avoir été enfant nous réhabilite, nous sauve, dans la mesure même où rien, en nous, ne peut le rappeler » (Gascar 6-7). 